\title{
17
}

\section{Reine Nervensache (Multiple Choice)}

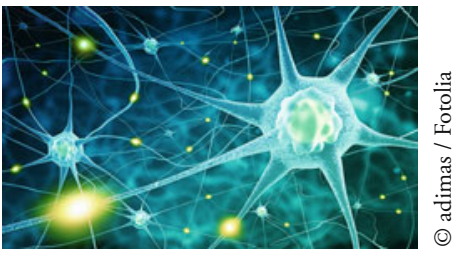

Wer wird denn hier die Nerven verlieren? Wähle die jeweils richtige Antwort aus und bilde das Lösungswort aus den erhaltenen Buchstaben.

1. Was ist eine Nervenfaser?
(M) Nervenbündel
(E) Axon
(T) Neuron

2. Aufgrund ihres Aussehens nennt man die Amygdala auch...
(E) Brücke
(R) Mandelkern
(A) Muschel

3. Um die Funktionsweise von Neuronen zu ergründen, bedient man sich sogenannter Riesenaxone. Sie entstammen...
(I) einem Wal
(N) dem Hausrind
(R) einem Kalmar

4. Nahe seinem Ende verzweigt sich das Axon in verdickte...
(S) Synapsen
(E) präsynaptische Endigungen
(N) Dendriten

5. Das sogenannte Ruhepotenzial von Nervenzellen wird bestimmt durch... (P) den Axon-Durchmesser (G) Natrium- und Kaliumionen (H) Hormone

6. Die Zeitspanne nach einem Aktionspotenzial, in welcher nach einem Aktionspotenzial kein Impuls mehr von der Nervenzelle erzeugt werden kann, nennt man...

(L) Referenzzeit (E) relative Refraktärzeit (U) absolute Refraktärzeit

7. Eine unwillkürliche, vom Zentralnervensystem gesteuerte Reaktion auf einen Reiz nennt man...
(N) Reflex
(D) Instinkt
(K) Rückreiz

8. Wodurch wird Tetanus - der Wundstarrkrampf - hervorgerufen?
(G) Bakterien
(A) psychologischer Schock
(O) ungeschützter Sex

Lösung:

(C) Springer-Verlag Berlin Heidelberg 2016

C. Reinbold, Fetthenne, Moderlieschen, Warzenbeißer,

DOI 10.1007/978-3-662-52817-4_17 